identify environmental and genetic factors associated with risk of vitamin D deficiency $(25[\mathrm{OH}] \mathrm{D}$ concentration $<50 \mathrm{nmol} / \mathrm{L})$.

Results Mean serum 25(OH)D concentration was $45.4 \mathrm{nmol} / \mathrm{L}$ (SD 25.3); 171/278 (61.5\%) participants were deficient. The following factors independently associated with increased risk of vitamin D deficiency: $\mathrm{BMI}>30 \mathrm{~kg} / \mathrm{m}^{2}(\mathrm{OR} 1.87, \mathrm{p}=0.04)$ and blood draw during winter and spring seasons (OR 3.00, p < 0.01 ; OR 2.50, $\mathrm{p}<0.01$, respectively). The following factors independently associated with reduced risk of deficiency: consumption of a vitamin D supplement, 100-400 IU/day (OR $0.42, \mathrm{p}<0.01)$; and a sunny holiday abroad no more than 2 months prior to blood draw (OR 0.27, $\mathrm{p}=0.02$ ). None of the 37 SNP investigated independently associated with vitamin D deficiency.

Conclusions Vitamin D deficiency was highly prevalent among COPD patients in this study. Obesity and winter and spring sampling were risk factors for deficiency. Recent travel to a sunny country and consumption of vitamin D supplements were protective. Genetic variants in the vitamin D pathway that have previously been shown to associate with risk of vitamin D deficiency in healthy adult populations were not associated with deficiency in this patient group.

\section{M146 VALIDATION OF FIVE NON-INVASIVE RESPIRATORY RATE MONITORS IN PATIENTS WITH COPD IN A LABORATORY SETTING}

${ }^{1}$ Noah Rubio, ${ }^{2}$ Brian McKinstry, ${ }^{3}$ Richard Parker, ${ }^{2}$ Hilary Pinnock, ${ }^{2}$ Christopher Weir, ${ }^{4}$ Janet Hanley, 'Claire Yerramasu, 'Leandro Cruz-Mantoani, 'William MacNee, 'Roberto A Rabinovich. 'Edinburgh Lung and the Environment Group Initiative (ELEGI), Centre for Inflammation and Research, Queens Medical Research Institute, Edinburgh, Edinburgh, UK; ${ }^{2}$ Centre for Population Health Sciences University of Edinburgh, Edinburgh, UK; ${ }^{3}$ Edinburgh Clinical Trial Unit, University of Edinburgh, Edinburgh, UK; ${ }^{4}$ Centre for Wellbeing and Healthcare, Edinburgh Napier University, Edinburgh, UK

\subsection{6/thoraxjnl-2014-206260.441}

Introduction There is a need of innovative models of care for patients with severe COPD and frequent AECOPD, and Telehealth $(\mathrm{TH})$ is part of these programs. But current systems are limited by the parameters feasibly monitored in a domestic setting and lack of a reliable method of predicting exacerbations. Evidence from hospital based studies show that breathlessness increases during exacerbations. If respiratory rate (RR) could be reliably monitored remotely it may provide a significant advance in predicting and identifying COPD exacerbations and monitoring recovery. The aim of this study is to validate five non-invasive RR monitors (M1 to M5) in patients with COPD in a laboratory setting against a gold standard measurement of RR.

Methods and results Five RR monitors identified in the literature were selected for validation against RR measured with a gold standard method (Oxycon mobile, Carefusion) in 23 patients with COPD (13 males, age $70 \pm 8.3$ years, FEV $_{1} 58.3$ $\pm 17.1 \%$ pred) during a $52 \mathrm{~min}$ protocol of a total of 19 activities of daily living (i.e sitting, standing, walking at different speeds, climbing stairs, lifting objects and sweeping the floor). Patients wore simultaneously the five monitors and the Oxycon mobile and RR was recorded breath by breath and averaged by minute. One minute of each activity was selected for analysis using Bland and Altman plots. Bias and limit of agreement (LoA) was established for each monitor (Figure 1). Bias and LoA for the five monitors were the following (M1 2.15 (-17.9 to 22.2), M2 3.1 (-8.7 to 14.9), M3 2.2 (-12.12 to 16.6), M4 -2.5 (-11.7
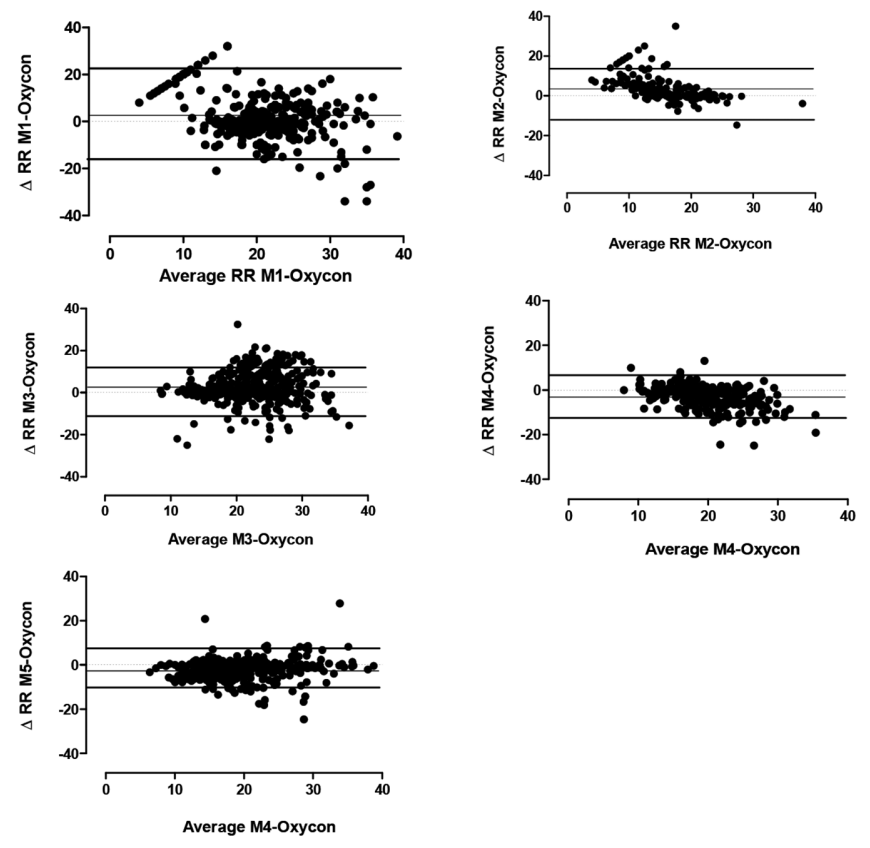

Abstract M146 Figure 1 Bland and Altman plots for RR between all five monitors and Oxycon Mobile

to 6.8) and M5 -1.9 (-10.8 to 6.9)). Patients were compliant with the use of the five monitors.

Conclusions Monitoring RR is feasible and non-intrusive in patients with COPD. We have identified two monitors (M4 and M5) with the lowest bias and the narrower LoA. These monitors will be further investigated in a home setting.

Funded by the Chief Scientific Office, CZH/4/826.

\section{M147 FEASIBILITY OF DELIVERING AN OCCUPATIONAL HEALTH INTERVENTION AIMED AT IMPROVING WORK PRODUCTIVITY, AMONG WORKING COPD PATIENTS}

K Kalirai, P Adab, R Jordan, JG Ayres, S Sadhra. The University of Birmingham, Birmingham, UK

\subsection{6/thoraxjnl-2014-206260.442}

Introduction There is evidence that workplace productivity may be impaired among working patients with COPD. Occupational health $(\mathrm{OH})$ interventions have been effective in improving work productivity in other chronic conditions. However, little is known about the feasibility and acceptability of such interventions among those with COPD.

Aim To assess feasibility and acceptability of an $\mathrm{OH}$ intervention in working COPD patients.

Methods Nested within a primary care COPD cohort $(\mathrm{n}=$ 1870), the study included all those who were in work ( $\mathrm{n}=$ 309). Eligible patients were invited for an interview and assessment with an $\mathrm{OH}$ practitioner. The aim was to explore and identify workplace factors that may contribute to their work performance or exacerbate their condition, and to suggest approaches to minimise any respiratory symptoms and improve work capability. Recommendations are sent to the patient, and with their permission, to their GP and employer. The acceptability of the intervention to employers will be explored as a separate part of the study.

Results Of those eligible, $43(13.9 \%)$ agreed to take part and 107 (34.6\%) declined. The most common reasons for declining 